\title{
Factores de riesgo asociados al aislamiento de Escherichia coli o Klebsiella pneumoniae productoras de betalactamasas de espectro extendido en un hospital de cuarto nivel en Colombia
}

\author{
Adriana Jiménez ${ }^{1}$, Alejandra Alvarado², Felipe Gómez², Germán Carrero², Claudia Fajardo³ \\ 1 Unidad de Prevención y Control de Infecciones, Hospital de San José, Facultad de Medicina, Fundación \\ Universitaria de Ciencias de la Salud, Bogotá, D.C., Colombia \\ 2 Servicio de Medicina Interna, Fundación Universitaria de Ciencias de la Salud, Bogotá, D.C., Colombia \\ 3 Área de Microbiología, Facultad de Medicina, Fundación Universitaria de Ciencias de la Salud, Bogotá, D.C., \\ Colombia
}

Introducción. Las betalactamasas de espectro extendido (BLEE) son un fenómeno de resistencia emergente de particular incidencia en América Latina. En Colombia existe poca información sobre los factores de riesgo asociados con su adquisición.

Objetivo. Determinar los factores de riesgo que están asociados a la infección o colonización por Escherichia coli o Klebsiella pneumoniae productoras de BLEE en pacientes mayores de 18 años.

Materiales y métodos. Se llevó a cabo un estudio de casos y controles con relación 1:1 en pacientes con aislamientos de E. coli o K. pneumoniae productoras de BLEE en cualquier tipo de muestra durante el periodo de enero de 2009 a noviembre de 2011 en el Hospital Universitario de San José.

Resultados. Se estudiaron 110 casos y 110 controles; $62,7 \%$ correspondió a E. coli y 37,3 \%, a K. pneumoniae. Como factores de riesgo independiente en el análisis multivariado se encontraron la insuficiencia renal crónica $\left(\mathrm{OR}=2,99 ; \mathrm{IC}_{95 \%}, 1,10-8,11 ; \mathrm{p}=0,031\right)$, la cirugía urológica $\left(\mathrm{OR}=4,78 ; \mathrm{IC}_{95 \%}\right.$, $1,35-16,87 ; p=0,015)$, el antecedente de uso de antibióticos en los tres meses anteriores $(O R=2,24$; $\left.I_{95 \%}, 1,09-4,60 ; p=0,028\right)$, el origen hospitalario de la infección $\left(O R=2,92 ; I_{95 \%}, 1,39-6,13 ; p=0,004\right)$ y la hospitalización previa (OR=1,59; $\left.I C_{95 \%}, 1,03-2,46 ; p=0,036\right)$.

Conclusión. Anticiparse al patrón de resistencia del microorganismo que infecta a un paciente con base en los factores de riesgo asociados permitiría la elección de un tratamiento antibiótico empírico apropiado, con el fin de lograr la disminución de la morbimortalidad de los pacientes.

Palabras clave: Escherichia coli, Klebsiella pneumoniae, factores de riesgo, betalactamasas, antibacterianos, farmacorresistencia microbiana.

doi: http://dx.doi.org/10.7705/biomedica.v34i0.1650

Risk factors associated with the isolation of extended spectrum betalactamases producing Escherichia coli or Klebsiella pneumoniae in a tertiary care hospital in Colombia

Introduction: Extended-spectrum beta-lactamases (ESBL) are an emerging resistance phenomenon with particular incidence in Latin America. In Colombia there is very little information regarding the risk factors associated with its acquisition.

Objective: To determine the risk factors that are associated with infection or colonization by Escherichia coli or Klebsiella pneumoniae producing ESBL in patients older than 18 years.

Materials and methods: A case-control study, ratio 1:1, in patients with an isolate from any sample of E. coli or K. pneumoniae producing ESBL in the period from January 2009 to November 2011 at San José University Hospital in Bogotá (Colombia).

Results: We studied 110 cases and 110 controls, 62,7\% were E. coli and 37,3\% K. pneumoniae. In the multivariate analysis the independent risk factors found were: chronic renal failure (odds ratio [OR] 2.99, confidence interval 95\% [95\% Cl] 1.10-8.11, $\mathrm{p}=0.031)$, urologic surgery $(\mathrm{OR} 4.7895 \% \mathrm{Cl}$ 1.35 to $16.87, p=0.015$ ), history of antibiotic use in the previous three months (OR $2.24,95 \% \mathrm{Cl} 1.09$ $-4.60, p=0.028)$, nosocomial origin of infection $(\mathrm{OR}=2.9295 \% \mathrm{Cl} 1.39-6.13, \mathrm{p}=0.004)$ and previous hospitalization (OR 1,59, 95\% Cl=1.03-2.46, $\mathrm{p}=0,036)$.

Conclusion: Anticipating the resistance pattern of the organism infecting a patient based on risk factors

\section{Contribución de los autores:}

Adriana Jiménez: investigadora principal, diseño del estudio, elaboración del artículo final.

Alejandra Alvarado, Felipe Gómez y Germán Carreño: diseño del estudio, recolección y análisis de los datos.

Claudia Fajardo: diseño del estudio, análisis microbiológico y selección de casos y controles.

Todos los autores aportaron al manuscrito final y conocen y aprueban su contenido final. 
may allow the choice of appropriate empirical antibiotic therapy, which will have an impact on reducing patients' morbidity and mortality.

Key words: Escherichia coli, Klebsiella pneumonia, risk factors, beta-lactamases anti-bacterial agents; drug resistance, microbial.

doi: http://dx.doi.org/10.7705/biomedica.v34i0.1650

En la década de los ochenta se inició el empleo clínico de las llamadas cefalosporinas de tercera generación, también conocidas como oximinocefalosporinas, las cuales eran resistentes a la acción de las betalactamasas circulantes en la época (SHV, TEM). Sin embargo, en 1982 se aisló en Alemania la primera betalactamasa (SHV-2) producida por Klebsiella pneumoniae capaz de hidrolizar estos antibióticos (1).

Este mecanismo de resistencia pronto se diseminó por todo el planeta; en 1989 se documentó la diseminación de una betalactamasa de espectro extendido (BLEE) de tipo CTX-M en Suramérica (2), cuya expansión fue de tal magnitud que la mayor prevalencia de BLEE en el mundo se informa hoy en Asia y América Latina, alcanzando en algunos países de esta última región una frecuencia de $48 \%$ para E. coliy de $60 \%$ para K. pneumoniae (3). En Colombia se desconoce la prevalencia global de las BLEE debido a que el programa nacional de vigilancia de la resistencia bacteriana se inició tan solo en el año 2012. En el Hospital San José la incidencia de BLEE para el periodo de estudio se estableció en $11,3 \%$ para $K$. pneumoniae y en $6,12 \%$ para E. coli.

La infección causada por estas bacterias se asocia con una mayor mortalidad (4) y, aunque los laboratorios de microbiología disponen de sistemas manuales y automatizados para identificar este fenómeno de resistencia, el anticiparse al resultado de los cultivos y determinar los factores de riesgo de infección por estos microorganismos en un paciente resulta fundamental para establecer el tratamiento empírico y las medidas de control adecuadas.

Varios estudios previos en diferentes latitudes han evaluado los factores de riesgo para la adquisición de estas bacterias, sin embargo, en Colombia solo se ha llevado a cabo un estudio en pacientes con infección de vías urinarias adquirida en la comunidad y no en infecciones en general $(5,6)$.

\section{Correspondencia:}

Adriana Jiménez, Calle 10 № 18-75, Bogotá, D.C., Colombia Teléfonos: (571) 3538000 , extensión 424, y (311) 811 8492; fax: (571) 3538000 , extensión 424

amjimenez@fucsalud.edu.co

Recibido: 15/05/13; aceptado: 08/08/13
El objetivo de este estudio fue determinar los factores de riesgo asociados a la infección o colonización por E. coli y K. pneumoniae productoras de BLEE para optimizar el tratamiento antibiótico empírico en pacientes infectados en cuyos aislamientos había una alta probabilidad de detectar bacterias con este mecanismo de resistencia, e instaurar oportunamente las medidas de control de infecciones.

\section{Materiales y métodos}

\section{Diseño del estudio}

Se realizó un estudio retrospectivo de casos y controles con relación 1:1 entre enero de 2009 y noviembre de 2011 en el Hospital San José de Bogotá, Colombia, hospital universitario de cuarto nivel de atención y 300 camas. Se calculó el tamaño de la muestra en 110 casos en relación $1: 1$ con los controles, para lo cual se empleó el programa estadístico StatsDirect, Version 2.7.2 (September, 2008). Se tuvo en cuenta el odds ratio $(\mathrm{OR})$ de menor magnitud encontrado en los estudios de factores de riesgo para infección por BLEE publicados hasta diciembre de 2008 , el cual correspondía a 2,7 y fue registrado por Mendelson para la variable de catéter urinario, con un error alfa de 0,05 y un poder de 0,8 (7).

\section{Método de laboratorio}

La identificación de las enterobacterias y la producción de BLEE se detectó mediante el equipo automatizado Micro Scan ${ }^{\circledR}$ según los criterios del Clinical and Laboratory Standards Institute (8).

\section{Selección de los casos}

El caso se definió como paciente mayor de 18 años con infección de cualquier origen y aislamiento de E. coli o K. pneumoniae productor de BLEE; solo se incluyó el primer aislamiento. Se emplearon los criterios de infección hospitalaria de los Centers for Disease Control and Prevention (CDC) (9).

\section{Selección de controles}

Los controles se definieron como pacientes con infección causada por las mismas enterobacterias no productoras de BLEE y fueron pareados por tipo de bacteria y origen de la muestra, y seleccionados 
en el mismo periodo de los casos de manera aleatoria empleando un método automatizado de generación de números aleatorios.

\section{Registro de datos}

Las variables demográficas se registraron en una base de datos; las enfermedades concomitantes que se contemplaron fueron la enfermedad pulmonar obstructiva crónica, la diabetes, la enfermedad cardiovascular, la insuficiencia renal crónica e, igualmente, los estados de inmunosupresión como el sida, las condiciones resultantes del empleo de inmunomoduladores o corticoides, la neutropenia, el cáncer, los antecedentes de cirugía, hospitalización y uso de antibióticos (cefalosporinas, carbapenémicos, piperacilinatazobactam, quinolonas, aminoglucósidos) en los tres meses anteriores y durante la hospitalización en la cual se identificó el aislamiento; también se consideró el origen hospitalario o comunitario de la infección, el área de hospitalización en el momento en que se realizó el cultivo y el uso de dispositivos médicos (respiración mecánica asistida, catéter vesical, catéter venoso central).

\section{Análisis estadístico}

El análisis estadístico se ejecutó con el programa Stata®, versión 10.0. Se estableció que los datos perdidos de las variables serían imputados empleando el promedio para las variables continuas y la moda para las discretas, y que las variables en las que se encontraran datos perdidos en más de $20 \%$ de las entradas serían eliminadas del modelo. El análisis bivariado para las variables categóricas se realizó mediante la prueba de ji al cuadrado o la prueba exacta de Fisher; para las variables continuas se empleó la t de Student. Las variables que obtuvieron un valor de $p<0,05$ en el análisis bivariado fueron introducidas en el análisis multivariado en un modelo de regresión logística. Los valores de $p$ fueron tomados a dos colas y se estableció el valor de $p<0,05$ como estadísticamente significativo.

\section{Consideraciones éticas}

No se requirió aprobación por parte del comité de ética institucional por tratarse de un estudio observacional retrospectivo en el que la información se extrajo de la historia clínica y los registros del laboratorio de microbiología, consignados como parte de la atención usual de los pacientes.

\section{Resultados}

Los 110 casos necesarios, según el cálculo del tamaño de la muestra, se recolectaron en un periodo de 35 meses, entre enero de 2009 y noviembre de 2011. De ellos, $62,7 \%$ presentó infección por $E$. coli y $37,3 \%$, por K. pneumoniae. Los controles se seleccionaron de manera aleatoria del mismo periodo. Entre los aislamientos, en $91,8 \%$ de los casos y $96,36 \%$ de los controles se consideró que había infección.

La edad media fue de 62,1 años para los casos (desviación estándar, DEะ17,99) y de 57,4 años (DE $\pm 19,81$ ) para los controles; $52,3 \%$ correspondió al sexo femenino y $47,7 \%$, al sexo masculino. Los aislamientos de cepas productoras de BLEE se originaron de las siguientes muestras: orina $(63,64 \%)$, herida quirúrgica $(11,8 \%)$, sangre $(10,9 \%)$, aspirado traqueal $(4,55 \%)$ y líquido peritoneal $(4,55 \%)$. Las características clínicas y demográficas, así como los resultados del análisis bivariado se presentan en el cuadro 1.

La enfermedad concomitante encontrada con mayor frecuencia en los casos y controles fue la de tipo cardiovascular (36,4 y 44,5\%, respectivamente), seguida de EPOC, diabetes e insuficiencia renal crónica (18 Vs. $9 \% ; p=0,049$ ). Entre los estados de inmunosupresión, el cáncer fue el más frecuente en los casos y controles (20,9 y $11,8 \%$, respectivamente).

El antecedente de hospitalización se encontró con mayor frecuencia en los casos que en los controles (27,27 Vs. $11,8 \%$; $p=0,015)$.

El origen hospitalario de la infección fue más frecuente en los casos (64,5\%) que en los controles (64,5 Vs. 41,8 \%; $p=0,007)$; no se encontró una diferencia estadísticamente significativa en cuanto al área de hospitalización (unidad de cuidados intensivos o pabellones) en que se encontraba el paciente en el momento en que se diagnosticó la infección, así como tampoco en el tiempo de hospitalización previo a dicho diagnóstico.

El uso de antibióticos previo a la infección (en una hospitalización pasada o en la del estudio) se identificó en la mayoría de casos y controles (81 y $70 \%$ ), aunque la diferencia no fue estadísticamente significativa. El antecedente de uso de antibióticos en los tres meses anteriores a la hospitalización fue mayor en los casos que en los controles (40 Vs. $21 \%$; $p=0,002)$; no se encontró una diferencia estadísticamente significativa en el empleo de antibióticos durante la hospitalización en la cual se detectó la infección (63\% en los casos Vs. 59 \% en los controles), a pesar de que, proporcionalmente, el uso de antibióticos durante la hospitalización 
Cuadro 1. Resultados del análisis univariado de factores de riesgo para aislamiento de BLEE

\begin{tabular}{|c|c|c|c|c|}
\hline \multirow{2}{*}{$\begin{array}{l}\text { Características } \\
\text { Edad, promedio }\end{array}$} & \multirow{2}{*}{$\begin{array}{c}\text { Casos } \\
\text { (n=110) positivos } \\
\text { para BLEE } n(\%)\end{array}$} & \multirow{2}{*}{$\begin{array}{c}\text { Controles } \\
\begin{array}{c}\text { (n= 110) negativos } \\
\text { para BLEE } n(\%)\end{array} \\
57,4( \pm 19.81)\end{array}$} & OR (IC95\%) & $\mathbf{p}$ \\
\hline & & & & 0,006 \\
\hline Sexo & & & & 0,13 \\
\hline Femenino & $52(47,27)$ & $63(57,27)$ & 1 & \\
\hline Masculino & $58(52,72)$ & $47(42,72)$ & $0,66(0,38-1,18)$ & \\
\hline \multicolumn{5}{|l|}{ Enfermedades concomitantes } \\
\hline Diabetes & $22(20,0)$ & $22(20,0)$ & $(0,48-2,04)$ & 1,00 \\
\hline Enfermedad pulmonar obstructiva crónica & $20(18,18)$ & $13(11,81)$ & $1,65(0,73-3,84)$ & 0,18 \\
\hline Enfermedad cardiovascular & $44(36,36)$ & $49(44,54)$ & $0,83(0,46-1,46)$ & 0,49 \\
\hline Insuficiencia renal crónica & $20(18,18)$ & $10(9,09)$ & $2,22(0,93-5,59)$ & 0,049 \\
\hline Urológicas & $15(13,63)$ & $10 \quad(9,09)$ & $1,57(0,63-4,13)$ & 0,28 \\
\hline Reumatológicas & $9(8,18)$ & $10(9,09)$ & $0,89(0,31-2,56)$ & 0,81 \\
\hline Neurológicas & $9(9,09)$ & $6(5,45)$ & $1,54(0,47-5,46)$ & 0,42 \\
\hline \multicolumn{5}{|l|}{ Inmunosupresión } \\
\hline Sida & $2(1,81)$ & $1(0,90)$ & $1,36(0,10-8,23)$ & 0,81 \\
\hline Inmunomoduladores & $7(6,36)$ & $8(7,27)$ & $0,86(0,25-2,84)$ & 0,78 \\
\hline Neutropenia & $3(2,72)$ & $3(2,72)$ & $0,99(0,12-7,56)$ & 0,99 \\
\hline Cáncer & $23(20,90)$ & $13(11,81)$ & $1,97(0,89-4,50)$ & 0,06 \\
\hline Tratamiento con corticoides & $8(7,27)$ & $8(7,27)$ & $1 \quad(0,31-3,18)$ & 1,00 \\
\hline \multicolumn{5}{|l|}{ Muestras } \\
\hline Orina & $70(63,64)$ & $70(63,64)$ & $(0,31-3,78)$ & 0,89 \\
\hline Herida quirúrgica & $13(11,8)$ & $13(11,8)$ & $(0,37-2,71)$ & 0,98 \\
\hline Sangre & $12(10,90)$ & $12(10,90)$ & $(0,33-3,36)$ & 0,92 \\
\hline Hospitalización previa † & $30(27,27)$ & $13(11,8)$ & $2,57(1,37-4,87)$ & 0,015 \\
\hline \multicolumn{5}{|l|}{ Origen de la infección } \\
\hline Comunidad & $39(35,45)$ & $64(58,18)$ & 1 & \\
\hline Hospital & $71(64,54)$ & $46(41,81)$ & $2,53(1,41-4,53)$ & 0,007 \\
\hline \multicolumn{5}{|l|}{ Área donde se diagnosticó la } \\
\hline infección hospitalaria & & & $1,34(0,56-, 18)$ & 0,462 \\
\hline Unidad de cuidados intensivos & $22(18,8)$ & $17(14,52)$ & & \\
\hline Pabellones & $49(41,8)$ & $29(24,7)$ & & \\
\hline Tiempo total de hospitalización (días) & & & $1,58(0,73-3,49)$ & 0,207 \\
\hline $0-15$ & $88(80)$ & $95(86,36)$ & & \\
\hline$>15$ & $22(20)$ & $15(13,62)$ & & \\
\hline \multicolumn{5}{|l|}{ Tiempo de hospitalización en unidad de } \\
\hline cuidados intensivos (días) & & & $1,34(0,563,18)$ & 0,175 \\
\hline $0-15$ & $102(92,72)$ & $105(95,45)$ & & \\
\hline$>15$ & $8(7,27)$ & $5(4,54)$ & & \\
\hline \multirow{2}{*}{\multicolumn{5}{|c|}{$\begin{array}{l}\text { Tiempo de hospitalización en pabellones } \\
\text { (días) }\end{array}$}} \\
\hline & & & $1,34(0,56-3,18)$ & 0,974 \\
\hline $0-15$ & $103(93,63)$ & $102(91,81)$ & & \\
\hline$>15$ & $7(6,36)$ & $8(7,27)$ & & \\
\hline Intervención quirúrgica & $52(47,27)$ & $31(28,18)$ & $2,28(1,25-4,16)$ & 0,003 \\
\hline Cirugía urológica & $21(19,09)$ & $4(3,63)$ & $6,25(1,99-25,79)$ & 0,000 \\
\hline Cirugía ortopédica & $7 \quad(6,36)$ & $1(0,90)$ & $7,40(0,91-33,68)$ & 0,030 \\
\hline Cirugía general & $19(17,27)$ & $19(17,27)$ & $1 \quad(0,46-2,14)$ & 1,00 \\
\hline Tratamiento antibiótico previo total & $89(80,9)$ & $77(70)$ & $1,81 \quad(0,92-3,59)$ & 0,060 \\
\hline En la hospitalización actual & $69(62,72)$ & $65(59)$ & $1,65(-0,67-2,00)$ & 0,58 \\
\hline En los 3 meses anteriores & $44(40)$ & $23(20,9)$ & $2,52(1,33-4,81)$ & 0,002 \\
\hline \multicolumn{5}{|l|}{ Dispositivos médicos } \\
\hline Respiración mecánica asistida & $19(0,17)$ & $18(0,16)$ & $1,06(0,49-2,3)$ & 0,857 \\
\hline Catéter vesical & $37(33,63)$ & $33(30)$ & $1,18 \quad(0,64-2,17)$ & 0,563 \\
\hline Catéter venoso central & $28(25,45)$ & $29(26,36)$ & $0,95(0,49-1,82)$ & 0,877 \\
\hline \multicolumn{5}{|l|}{ Aislamiento } \\
\hline Escherichia coli & $69(62,72)$ & $69(62,72)$ & $(0,40-2,67)$ & 0,93 \\
\hline Klebsiella pneumoniae & $41(37,27)$ & $41(37,27)$ & $(0,35-2,47)$ & 0,90 \\
\hline
\end{tabular}

† En los tres meses anteriores a la hospitalización 
fue mayor comparado con el uso de antibióticos en los tres meses anteriores. Los antibióticos más empleados antes del aislamiento fueron piperacilinatazobactam (22,7 \%), carbapenémicos $(12,72 \%)$, cefalosporinas de tercera generación (10\%), y quinolonas $(6,3 \%)$.

La intervención quirúrgica se detectó con mayor frecuencia en los casos que en los controles (47,3 Vs. $28,2 \%, p=0,003)$; la cirugía urológica fue la más frecuente en los casos ( 19 Vs. $3 \%$; $p=0,000$ ); en segundo lugar se ubicaron los procedimientos de cirugía general con una distribución homogénea en los casos y controles (17,3\% para ambos); el tercer lugar lo ocupó la cirugía ortopédica, también más frecuente en los casos que en los controles (6,36 Vs. 0,9\%; $p=0,003)$.

En el análisis multivariado (cuadro 2), empleando un modelo de regresión logística, se encontró que la insuficiencia renal crónica, el origen hospitalario de la infección, el antecedente de hospitalización o el empleo de antibióticos en los tres meses anteriores y la cirugía urológica alcanzaron la significación estadística, por lo que se consideraron factores de riesgo independientes para la adquisición de $E$. coli o K. pneumoniae productoras de BLEE.

\section{Discusión}

Entre los estudios de factores de riesgo publicados, la variable que se ha encontrado con mayor frecuencia ( $42 \%$ de los estudios) es el antecedente de uso de antibióticos (5), factor de riesgo independiente también identificado en este estudio. No se encontró asociación estadísticamente significativa con ningún antibiótico en particular, aunque el antibiótico usado con mayor frecuencia (22 \%) fue piperacilina-tazobactam, mientras que el antecedente de uso de quinolonas solo se estableció en $6 \%$ de los pacientes. A pesar de que el antecedente de uso de cefalosporinas de tercera generación y quinolonas son factores de riesgo ampliamente reconocidos, el uso de piperacilinatazobactam también ha sido asociado con la selección de BLEE $(10,11)$.

El uso de catéter urinario es el tercero en frecuencia entre los factores de riesgo registrados en los estudios sobre dicho aspecto $(7,12-14)$; sin embargo, en este estudio no se encontró asociación con esta variable ni con la respiración mecánica asistida, que también ha sido asociada con la adquisición de BLEE en otros estudios $(15,16)$.

El origen hospitalario de la infección como factor de riesgo independiente, también encontrado en esta investigación, ha sido asociado en, por lo menos, cinco estudios más, lo que ratifica la importancia de la vigilancia de las infecciones hospitalarias y la implementación temprana de las precauciones de contacto para disminuir el riesgo de transmisión cruzada de estos microorganismos $(13,17-20)$.

En cuanto a las enfermedades concomitantes, a pesar de que siempre son variables exploradas en los diferentes estudios, sólo el de Cheol In Kang (21) encontró asociación con la insuficiencia hepática y el de Mendelson (7), con la anemia. Este estudio es el primero en encontrar asociación con la insuficiencia renal crónica y con la cirugía urológica, lo que podría explicarse por el hecho de que nuestro hospital es un centro de referencia para la atención de pacientes con enfermedades de las vías urinarias. Previamente, en el estudio de Rodríguez Baño (13), se había encontrado asociación con la enfermedad obstructiva de las vías urinarias que, en muchos casos, es la causa subyacente de la intervención quirúrgica.

La asociación con la hospitalización previa, que también ha sido informada en otros estudios, debe interpretarse con cautela, ya que quizá la causa esté más relacionada con la exposición previa a antibióticos o con la intervención quirúrgica.

Cuadro 2. Resultados del análisis multivariado de los factores de riesgo para aislamiento de BLEE

\begin{tabular}{|c|c|c|c|c|c|}
\hline \multirow[t]{2}{*}{ Factores de riesgo } & $\begin{array}{l}\text { Casos positivos } \\
\text { para BLEE }\end{array}$ & $\begin{array}{c}\text { Controles negativos } \\
\text { para BLEE }\end{array}$ & \multirow[t]{2}{*}{ OR } & \multirow[t]{2}{*}{ IC $_{95 \%}$} & \multirow[t]{2}{*}{$\mathbf{p}$} \\
\hline & $\begin{array}{l}n=110 \\
n(\%)\end{array}$ & $\begin{array}{l}n=110 \\
n(\%)\end{array}$ & & & \\
\hline Insuficiencia renal crónica & $20(18,18)$ & $10(9,09)$ & 2,99 & $(1,10-8,11)$ & 0,031 \\
\hline Cirugía urológica & $21(19,09)$ & $4(3,63)$ & 2,24 & $(1,35-16,8)$ & 0,015 \\
\hline Antibiótico previo* & $44(40)$ & $23(20,90)$ & 2,24 & $(1,09-4,60)$ & 0,028 \\
\hline Origen de la infección hospitalaria & $71(64,54)$ & $46(41,81)$ & 2,92 & $(1,39-6,13)$ & 0,004 \\
\hline Hospitalización previa & $30(27,27)$ & $13(11,8)$ & 1,59 & $(1,03-2,46)$ & 0,036 \\
\hline
\end{tabular}

${ }^{*}$ En los tres meses anteriores a la hospitalización 
Hasta donde sabemos, este es el primer estudio en América Latina que evalúa factores de riesgo para la infección y colonización de cualquier sitio anatómico por este tipo de bacterias. Un estudio anterior de Leal, et al. (6), realizado en hospitales colombianos, se centró en la búsqueda de factores de riesgo para la infección de las vías urinarias de origen comunitario por enterobacterias resistentes a cefalosporinas de tercera generación, y el estudio de Superti, et al., realizado en Brasil, investigó factores de riesgo asociados a la bacteriemia (22).

Entre las limitaciones del estudio debe considerarse que dada la naturaleza retrospectiva del diseño, es posible que algunas variables no quedaran consignadas en las historias clínicas de los pacientes, así como el hecho de que fue realizado en un solo centro asistencial de orientación quirúrgica, lo que limita su extrapolación a otras instituciones.

El conocimiento de los factores de riesgo asociados a este fenómeno de resistencia permite el inicio del tratamiento antimicrobiano empírico pertinente y la instauración de las medidas de control oportunas. Se requieren más estudios de este tipo con una muestra mayor que permita construir una escala de riesgo.

\section{Conflicto de intereses}

Todos los autores declaran no tener conflicto de intereses en relación con este estudio.

\section{Financiación}

Este estudio fue financiado en su totalidad por la Fundación Universitaria de Ciencias de la Salud.

\section{Referencias}

1. Klee C, Nies BA, Meyer JF, Tolxdorff-Neutzling RM, Wiedemann B. Evolution of plasmid-coded resistance to broad-spectrum cephalosporins. Antimicrob Agents Chemother. 1985;28:302-7. http://dx.doi.org/10.1128/AAC. 28.2.302

2. Radice M, Power P, Di Conza J, Gutkind G. Early dissemination of CTX-M-derived enzymes in South America. Antimicrob Agents Chemother. 2002;46:602-4. http://dx.doi. org/10.1128/AAC.46.2.602-604.2002

3. Gales AC, Castanheira M, Jones RN, Sader HS. Antimicrobial resistance among Gram-negative bacill isolated from Latin America: Results from SENTRY Antimicrobial Surveillance Program (Latin America, 20082010). Diagn Microbiol Infect Dis. 2012;73:354-60. http://dx doi.org/10.1016/j.diagmicrobio.2012.04.007

4. Schwaber MJ, Carmeli Y. Mortality and delay in effective therapy associated with extended-spectrum B-lactamase production in Enterobacteriaceae bacteraemia: A systematic review and meta-analysis. J Antimicrob Chemother. 2007; 60:913-20. http://dx.doi.org/10.1093/jac/dkm318
5. Jiménez A, Forero G. Factores de riesgo en infección y colonización por Escherichia coli y Klebsiella pneumoniae productoras de betalactamasas de espectro extendido. Repertorio de Medicina y Cirugía. 2013;22:10-20.

6. Leal A, Cortés J, Arias G, Ovalle MV, Saavedra S, Buitrago G, et al. Emergencia de fenotipos resistentes a cefalosporinas de tercera generación en Enterobacteriaceae causantes de infección del tracto urinario de inicio comunitario en hospitales de Colombia. Enferm Infecc Microbiol Clin. 2013;31:298-303. http://dx.doi.org/10.1016/j.eimc. 2012. 04.007

7. Mendelson G, Hait V, Ben-Israel J, Gronich D, Granot E, Raz R. Prevalence and risk factors of extendedspectrum beta-lactamase-producing Escherichia coli and Klebsiella pneumoniae in an Israeli long-term care facility. Eur J Clin Microbiol Infect Dis. 2005;24:17-22. http://dx.doi. org/10.1007/s10096-004-1264-8

8. Clinical and Laboratory Standards Institute/NCCLS. Performance standards for antimicrobial susceptibility testing; nineteenth informational supplement. Wayne, PA: Clinical and Laboratory Standards Institute; 2009

9. Horan TC, Andrus M, Dudeck MA. CDC/NHSN surveillance definition of health care-associated infection and criteria for specific types of infections in the acute care setting. Am $\mathrm{J}$ Infect Control. 2008;36:309-32. http://dx.doi.org/10.1016/j. ajic.2008.03.002

10. Bermejo J, Bencomo B, Arnesi N, Lesnaberes $\mathbf{P}$, Borda N, Notario R. Alta correlación entre el consumo de ciprofloxacina y la prevalencia de Klebsiella pneumoniae productora de $\beta$-lactamasas de espectro extendido. Rev Chil Infect. 2006;23:316-20. http://dx.doi.org/10.4067/S071610182006000400004

11. Han JH, Kasahara K, Edelstein PH, Bilker WB, Lautenbach E. Risk factors for infection or colonization with CTX-M extended-spectrum- $\beta$-lactamase-positive Escherichia coli. Antimicrob Agents Chemother. 2012;56:5575-80. http://dx. doi.org/10.1128/AAC.01136-12

12. Wu UI, Yang CS, Chen WC, Chen YC, Chang SC. Risk factors for bloodstream infections due to extended-spectrum beta-lactamase-producing Escherichia coli. J Microbiol Immunol Infect. 2010;43:310-6. http://dx.doi.org/10.1016/ S1684-1182(10)60048-5

13. Rodríguez-Baño J, Picón E, Gijón P, Hernández JR, Ruiz M, Peña C, et al. Community-onset bacteremia due to extended-spectrum beta-lactamase-producing Escherichia coli: risk factors and prognosis. Clin Infect Dis. 2010;50:40-8. http://dx.doi.org/10.1086/649537.

14. Rodríguez-Baño J, Navarro MD, Romero L, Muniain MA, Cueto MD, Gálvez J, et al. Risk-factors for emerging bloodstream infections caused by extended-spectrum betalactamase-producing Escherichia coli. Clin Microbiol Infect. 2008;14:180-3.http://dx.doi.org/10.1111/j.1469-0691.2007. 01884.x

15. Chaiwarith R, Pasogpakdee P, Salee $P$, Kanjanaratanakorn K, Sirisanthana T, Supparatpinyo K. Risk factors for extended-spectrum beta-lactamase-producing Klebsiella pneumoniae and Escherichia coli acquisition in a tertiary care teaching hospital in Thailand. J Hosp Infect. 2009: 285-6. http://dx.doi.org/10.1016/j.jhin.2008.11.021

16. Kuster SP, Hasse B, Huebner V, Bansal V, Zbinden R, Ruef $\mathbf{C}$, et al. Risks factors for infections with extended- 
spectrum beta-lactamase-producing Escherichia coli and Klebsiella pneumoniae at a tertiary care university hospital in Switzerland. Infection. 2010;38:33-40. http://dx.doi.org/ 10.1007/s15010-009-9207-z

17. Kang Cl, Song JH, Chung DR, Peck KR, Ko KS, Yeom JS, et al. Risk factors and treatment outcomes of communityonset bacteraemia caused by extended-spectrum betalactamase-producing Escherichia coli. Int J Antimicrob Agents. 2010;36:284-7. http://dx.doi.org/10.1016/j.ijantimicag. 2010.05.009

18. Kang Cl, Chung DR, Ko KS, Peck KR, Song JH. Risk factors for infection and treatment outcome of extendedspectrum beta-lactamase-producing Escherichia coli and Klebsiella pneumoniae bacteremia in patients with hematologic malignancy. Ann Hematol. 2012;91:115-21. http://dx. doi.org/10.1007/s00277-011-1247-7

19. Ho PL, Chan WM, Tsang KW, Wong SS, Young K. Bacteremia caused by Escherichia coli producing extendedspectrum beta-lactamase: A case-control study of risk factors and outcomes. Scand J Infect Dis. 2002;34:567-73.
20. García Hernández A, García-Vázquez E, Gómez Gómez J, Canteras M, Hernández-Torres A, Ruiz Gómez J. Predictive factors of ESBL versus non-ESBL Escherichia coli bacteraemia and influence of resistance on the mortality of the patients. Med Clin (Barc). 2011;136:56-60. http://dx. doi.org/10.1016/j.medcli.2010.05.014

21. Kang Cl, Song JH, Chung DR, Peck KR, Ko KS, Yeom JS, et al. Risk factors and treatment outcomes of community-onset bacteraemia caused by extendedspectrum beta-lactamase-producing Escherichia coli. Int J Antimicrob Agents 2010;36:284-7. http://dx.doi.org/10.1016/j. ijantimicag.2010.05.009

22. Superti SV, Augusti G, Zavascki AP. Risk factors for and mortality of extended-spectrum-beta-lactamaseproducing Klebsiella pneumoniae and Escherichia coli nosocomial bloodstream infections. Rev Inst Med Trop Sao Paulo. 2009;51:211-6. http://dx.doi.org/10.1590/S003646652009000400006 